Acta Mathematica Academiae Paedagogicae Nyíregyháziensis

17 (2001), 81-96

www.emis.de/journals

\title{
DIOPHANTINE PROPERTIES OF LINEAR RECURSIVE SEQUENCES II
}

\author{
ATTILA PETHŐ
}

Dedicated to the 60th birthday of Professor Árpád Varecza

\begin{abstract}
Let $G_{n}$ be a linear recursive sequence of integers and $P(y)$ be a polynomial with integer coefficients. In this paper we are given a survey on results on the solutions of diophantine equation $G_{n}=P(y)$. We prove especially that if $G_{n}$ is of order three such that its characteristic polynomial is irreducible and has a dominating root then there are only finitely many perfect powers in $G_{n}$.
\end{abstract}

\section{INTRODUCTION}

Let $G_{n}$ be a $k$-th $(k \geq 2)$ order linear recursive sequence, in the sequel LRS, of integers defined by initial terms $G_{0}, \ldots, G_{k-1} \in \mathbb{Z}$ and by the relation

$$
G_{n+k}=A_{1} G_{n+k-1}+\cdots+A_{k} G_{n}
$$

for $n \geq 0$, where $A_{1}, \ldots, A_{k} \in \mathbb{Z}, A_{k} \neq 0$. We assume that at least one of the initial terms of $G_{n}$ is non-zero. In the present paper we continue our survey on results concerning the mixed exponential-polynomial diophantine equation

$$
G_{n}=P(y),
$$

where $P(y) \in \mathbb{Z}[y]$ denotes a polynomial of degree $d \geq 2$. In the first part [P6] we concentrated on results proved by using elementary and algebraic tools. Here we are dealing with applications of lower bounds for linear forms in logarithms of algebraic numbers.

Most of the text of the paper is four years old. In 1996 I delivered an invited talk at the Seventh International Research Conference on Fibonacci Numbers and Their Applications. The edited version of the first part of that talk appeared in [P6], while the second part remains up to now unpublished. I extended this manuscript with some new results.

Although the paper is a survey it includes new results too. You find an explicit bound for the exponent of perfect powers appearing in the classical Fibonacci and Lucas sequences. Another new result is Theorem 6, where we prove by the combination of effective and non-effective methods that, under certain condition, there exit only finitely many perfect powers in cubic recurrences.

2000 Mathematics Subject Classification. 11D61, 11D25.

Key words and phrases. Linear recursive sequence, characteristic polynomial. Linear forms in logarithms of algebraic numbers, subspace theorem.

This paper was written when the author was a visiting professor at the Mathematical Institute of the Technical University of Graz, Austria.

Research partially supported by Hungarian National Foundation for Scientific Research Grant No 16741 and 25157. 


\section{ApPlications of LOWER BOUNDS FOR LINEAR FORMS IN LOGARITHMS OF ALGEBRAIC NUMBERS}

The most powerful tools for the solution of equation (1) are certainly lower bounds for linear forms in logarithms of algebraic numbers. One can prove not only general finiteness theorems, but combining with numerical diophantine approximation techniques one can completely solve single and even parametrized families of equations. In this section we collect some result proved on this way.

The first general lower bound for linear forms in logarithms of algebraic numbers was proved by A. Baker [Ba1]. Since them several improvements and refinements appeared in the literature. It has a lot of applications. Analogous results were proved for linear forms in p-adic, in elliptic and in p-adic elliptic logarithms of algebraic numbers as well. Although they have also important applications in the theory of recurrences, see [ShT], we are dealing here only with the most classical case. For a collection of results proved by Baker's method and further references we refer to the monographs [Ba2, ShT, Sm].

Here we cite three recent results of this kind. All three are very sharp, but under certain circumstances they give different results (sometimes nothing), therefore it is worth to choose the best appropriate one in a concrete case. We need some further definition. Let the algebraic number $\beta$ be a zero of the irreducible polynomial $p(y)=a_{t} y^{t}+\cdots+a_{0} \in \mathbb{Z}[y]$, where $\left(a_{t}, \ldots, a_{0}\right)=1$. Denote $\beta=\beta_{1}, \ldots, \beta_{t}$ the zeros of $p(y)$. The absolute logarithmic height of $\beta$ is defined by

$$
h(\beta)=\frac{1}{t} \log \left(\prod_{i=1}^{t} \max \left\{1,\left|\beta_{i}\right|\right\}\right) .
$$

In the sequel let $\alpha_{1}, \ldots, \alpha_{m}$ be non-zero algebraic numbers and $b_{1}, \ldots, b_{m}$ rational integers. For $i=1, \ldots, m$, let $\log \alpha_{i}$ be a determination of the logarithm of $\alpha_{i}$ and

$$
\Lambda=b_{1} \log \alpha_{1}+\cdots+b_{m} \log \alpha_{m} .
$$

Assume further that $\Lambda \neq 0$.

The first theorem is due to Baker and Wüstholz [BW].

Theorem 1. Let $D=\left[\mathbb{Q}\left(\alpha_{1}, \ldots, \alpha_{m}\right): \mathbb{Q}\right]$ and $B=\max \left\{\left|b_{1}\right|, \ldots,\left|b_{m}\right|, e\right\}$. Then

$$
\log |\Lambda| \geq-18(m+1) ! m^{m+1}(32 D)^{m+2} h\left(\alpha_{1}\right) \cdots h\left(\alpha_{m}\right) \log B .
$$

This theorem is very easy to apply, but it makes not possible for example to prove a bound for exponents for perfect powers in recursive sequences. For such purpose we shall apply the following result of Waldschmidt [W].

Theorem 2. Put

$$
D=\left[\mathbb{Q}\left(\alpha_{1}, \ldots, \alpha_{m}\right): \mathbb{Q}\right] \quad \text { and } \quad g=\left[\mathbb{R}\left(\log \alpha_{1}, \ldots, \log \alpha_{m}\right): \mathbb{R}\right] .
$$

Let $h_{1}, \ldots, h_{m}, E$ and $f$ be positive real numbers such that

$$
\log h_{i} \geq h\left(\alpha_{i}\right), \quad(1 \leq i \leq m), \quad h=\max \left\{h_{1}, \ldots, h_{m}\right\}
$$

and

$$
e \leq E \leq \min \left\{h_{1}^{D}, \ldots, h_{m}^{D}, \frac{m D}{f}\left(\sum_{i=1}^{m} \frac{\left|\log \alpha_{i}\right|}{\log h_{i}}\right)^{-1}\right\} .
$$

Assume that $b_{m} \neq 0$ and put

$$
\begin{gathered}
B=\max _{1 \leq j \leq m}\left\{\frac{\left|b_{m}\right|}{\log h_{j}}+\frac{\left|b_{j}\right|}{\log h_{m}}\right\}, \\
Z_{0}=\max \left\{7+3 \log m, \frac{g}{D} \log E, \log \left(\frac{D}{\log E}\right)\right\}, \quad H_{0}=\max \left\{4 m Z_{0}, \log B\right\}
\end{gathered}
$$


and

$$
U_{0}=\max \left\{D^{2} \log h, D^{m+2} H_{0} Z_{0} \log h_{1} \cdots \log h_{m}(\log E)^{-m-1}\right\} .
$$

Then the linear form $\Lambda$ satisfies

$$
|\Lambda| \geq \exp \left\{-1500 g^{-m-2} 2^{2 m} m^{3 m+5}\left(1+\frac{g}{f}\right)^{m} U_{0}\right\} .
$$

In solving equations related to second order recurrences we often meet cases, when only two algebraic numbers are involved, i.e. $m=2$. Then there are even sharper lower bounds available. We cite here Corrollaire 1 of Laurent, Mignotte and Nesterenko [LMN].

Theorem 3. Let $\alpha_{1}, \alpha_{2}$ be multiplicatively independent algebraic numbers and $b_{1}, b_{2}$ be integers. Put $D=\left[\mathbb{Q}\left(\alpha_{1}, \alpha_{2}\right): \mathbb{Q}\right] /\left[\mathbb{R}\left(\alpha_{1}, \alpha_{2}\right): \mathbb{R}\right]$ and

$$
\Lambda=b_{1} \log \alpha_{1}-b_{2} \log \alpha_{2} .
$$

Let $h_{1}, h_{2}>1$ real numbers satisfying

and put

$$
\log h_{i} \geq \max \left\{h\left(\alpha_{i}\right), \frac{\left|\log \alpha_{i}\right|}{D}, \frac{1}{D}\right\}, i=1,2
$$

Then

$$
b=\frac{b_{1}}{D \log h_{2}}+\frac{b_{2}}{D \log h_{1}} .
$$

$$
\log |\Lambda| \geq-30.9 D^{4}\left(\max \left\{\log b, \frac{21}{D}, \frac{1}{2}\right\}\right)^{2} \log h_{1} \log h_{2} .
$$

In the applications of the above lower bounds the following simple lemmata are useful. For their proofs we refer to [PW].

Lemma 1. Let $a \in \mathbb{R}$. If $0<a<1$ and $0<|x|<a$ then

$$
|\log (1+x)|<\frac{-\log (1-a)}{a}|x| .
$$

Lemma 2. Let $a \geq 0, h \geq 1, b>\left(e^{2} / h\right)^{h}$, and let $x \in \mathbb{R}, x>1$ satisfy

$$
x \leq a+b \log ^{h} x .
$$

Then

$$
x<2^{h}\left(a^{1 / h}+b^{1 / h} \log \left(h^{h} b\right)\right)^{h} .
$$

2.1. Perfect powers in LRS. Bounding the exponents. Let $G_{n}$ be a LRS with characteristic polynomial $C_{G}(y)=y^{k}-A_{1} y^{k-1}-\cdots-A_{k}$. Let $\alpha_{1}, \ldots, \alpha_{h}$ denote the distinct zeros of $C_{G}$ with multiplicities $m_{1}, \ldots, m_{h}$ respectively. Then there exist polynomials $g_{i}(y) \in \mathbb{Q}\left(\alpha_{1}, \ldots, \alpha_{h}\right)[y]$ of degree less than $m_{i}, i=1, \ldots, h$, such that

$$
G_{n}=g_{1}(n) \alpha_{1}^{n}+\cdots+g_{h}(n) \alpha_{h}^{n}
$$

holds for any integer $n \geq 0$.

In this section we are dealing with the solutions $n, y, q \in \mathbb{Z}$ of the equation

$$
G_{n}=y^{q} .
$$

The letters $c_{1}, c_{2}, \ldots$ will denote effectively computable constants depending only on the initial terms and on the coefficients of the characteristic polynomial of $G_{n}$.

For binary recurrences Shorey and Stewart [ShSt1] and independently the author of the present notes [P1] proved that $\max \{n,|y|, q\}<c_{1}$. We come back to this result in section 2.3. For higher order recurrences much less is known. There are results only in that case, when $G_{n}$ has a dominating characteristic zero. Under this assumption Shorey and Stewart [ShSt1] proved that $q<c_{2}$ provided $|y|>1$. 
Moreover Nemes and Pethő [NP1] showed that if $T(y) \in \mathbb{Z}[y]$ is a fixed polynomial, then $q<c_{3}$ holds for any solutions $n, y, q \in \mathbb{Z}$ of the equation

$$
G_{n}=y^{q}+T(y)
$$

as well, provided $|y|>1$. The constant $c_{3}$ depends naturally on the coefficients of $T(y)$ too. Recently Kiss [K3] succeeded to refine in some cases this result. He also proved [K1] the lower bound $\left|G_{n}-y^{q}\right|>e^{c n}$, provided $\alpha_{1}>\left|\alpha_{2}\right|>\left|\alpha_{i}\right|, i>2$. Shorey and Stewart [ShSt2] established under the same assumption that there exist only finitely many solution $n, y, q$ with $n \geq 0,|y|>1$ and

$$
q>\max \left\{\frac{k \log \alpha_{1}}{\log \left(\alpha_{1} / \max \left\{1,\left|\alpha_{2}\right|\right\}\right)},\left[\mathbb{Q}\left(\alpha_{1}\right): \mathbb{Q}\right]+\operatorname{deg} T\right\} .
$$

We also mention that this result is not effective, we do not know any upper bound for $\max \{n,|y|\}$. A collection of the most general results of this kind you find in $[\mathrm{ShT}]$.

Remark that for fixed $q$ the last equation may have infinitely many integer solution. Indeed, let $H_{n}$ be a $\operatorname{LRS}$ and $T(y) \in \mathbb{Z}[y]$ a polynomial. Then $G_{n}=T\left(H_{n}\right)$ is an LRS as well and the equation

$$
G_{n}=T(y)
$$

has infinitely many solutions. It is widely believed that the converse is also true, i.e. the equation $G_{n}=T(y)$ has infinitely many solution only when $G_{n}$ has a subsequence of the form $T\left(H_{n}\right)$, with an LRS $H_{n}$. Positive answer for this problem was known until very recently again only for binary recurrences and only in the case when $\left|A_{2}\right|=1$. (See [NP2, P4].) The situation changed with the papers [CZ1] and [CZ2]. In that papers Corvaja and Zannier proved similar results under quite general conditions. Let $G_{n}$ be an LRS such that all of the zeros of its characteristic polynomial are simple and such that $\alpha_{1}$ is dominating, i.e. $1 \neq\left|\alpha_{1}\right|>\left|\alpha_{i}\right|, i=$ $2, \ldots, k$. Let $T(y) \in \mathbb{Z}[y]$ be a monic polynomial. If the equation (5) has infinitely many $n, y \in \mathbb{Z}, n \geq 0$ solutions then there exists an LRS $H_{n}$ of algebraic numbers and an arithmetical progression $P$ such that $G_{n}=T\left(H_{n}\right)$ holds for all $n \in P$. Especially, let $q \geq 2$ be fixed and let $G_{n}$ be a $q$-th power for infinitely many $n$, then $G_{n}=H_{n}^{q}$ holds for all $n$ belonging to an arithmetical progression. In fact the theorem of Corvaja and Zannier is much more general.

In the above mentioned papers the dependence on the constants of the parameters was never examined. In this section, following essentially the earlier line, I intend to prove an explicit upper bound for the exponents of possible perfect powers in $G_{n}$.

Theorem 4. Let $G_{n}$ be a LRS such that all of the zeros of its characteristic polynomial are simple and $\alpha:=\alpha_{1}>\left|\alpha_{2}\right| \geq\left|\alpha_{i}\right|, i=3, \ldots, k$. Put

$$
\delta:=\frac{\log \left|\alpha_{2}\right|}{\log \alpha}, \quad c_{1}:=\sum_{i=2}^{k} \frac{\left|g_{i}\right|}{\left|g_{1}\right|}, \quad a_{1}=\exp \left\{\max \left\{h\left(g_{1}\right), e\right\}\right\}
$$

and

$$
\begin{aligned}
c_{2}:= & \max \left\{\frac{1}{\log 2}\left(\frac{\log \left(4 c_{1}\right)}{1-\delta}+\log \left(c_{1}+1\right)+\log \left|g_{1}\right|\right), \frac{\log \alpha}{2}(k !)^{14},\right. \\
& 3 \log \left(\frac{5\left|g_{1}\right|}{4}\right)+\frac{2 \log \left(1.15 c_{1}\right)}{1-\delta}+ \\
& \left.\frac{1}{1-\delta} 4 \cdot 10^{13}(k !)^{7} \log a_{1} \log \alpha\left(217 \log (k !) \log \log a_{1}-\log (1-\delta)\right)\right\} .
\end{aligned}
$$


Assume that $n, y, q \in \mathbb{Z}$ is a solution of (3) such that $|y|>1$. Then

$$
q<c_{2}
$$

Proof. Let $\mathbb{K}=\mathbb{Q}\left(\alpha_{1}, \ldots, \alpha_{k}\right)$. First we remark, that as the zeros of $C_{G}(y)$ are simple, the polynomials $g_{i}(y) \in \mathbb{K}[y], i=1, \ldots, k$ are actually constants, thus the definition of $a_{1}, c_{1}$ and $c_{2}$ is correct.

Let $n, y, q \in \mathbb{Z}$ be a solution of (3) such that $|y|>1$ and

$$
q \geq \frac{1}{\log 2}\left(\frac{\log \left(4 c_{1}\right)}{1-\delta}+\log \left(c_{1}+1\right)+\log \left|g_{1}\right|\right) .
$$

Then

$$
\left|g_{1}\right|\left(c_{1}+1\right)\left(4 c_{1}\right)^{1 /(1-\delta)} \leq 2^{q} \leq|y|^{q}<\left|g_{1}\right|\left(c_{1}+1\right) \alpha^{n}
$$

thus $\alpha^{n(1-\delta)}>4 c_{1}$. We now obtain the following important inequality

$$
\left|\frac{|y|^{q}}{\left|g_{1}\right| \alpha^{n}}-1\right| \leq \sum_{i=2}^{k}\left|\frac{g_{i}}{g_{1}}\right|\left|\frac{\alpha_{i}}{\alpha}\right|^{n} \leq c_{1} \alpha^{-n(1-\delta)}<\frac{1}{4} .
$$

From this we obtain on the one hand

$$
\frac{q}{\log \alpha}-\frac{\log \left(5\left|g_{1}\right| / 4\right)}{\log \alpha \log |y|}<\frac{n}{\log |y|}<\frac{q}{\log \alpha}-\frac{\log \left(3\left|g_{1}\right| / 4\right)}{\log \alpha \log |y|}
$$

and

$$
\left|\Lambda_{1}\right|:=|\log | g_{1}|+n \log \alpha-q \log | y||<1.15 \cdot c_{1} \alpha^{-n(1-\delta)}
$$

on the other hand. The last inequality follows from Lemma 1. The expression staying on the left hand side of (7) is a linear form in logarithms of algebraic numbers and we have just proved a sharp upper bound for it.

A useful lower bound can be derived in this case only from Theorem 2 . We have in the present case $m=3, g=1, b_{1}=1, b_{2}=n, b_{3}=q$ and $D \leq k$ !, but actually we need only an upper bound for the degree of $\mathbb{K}$ over $\mathbb{Q}$, therefore we may take $D=k !$. We choose $E=e, f=1, h_{1}=a_{1}, \log h_{2}=k \log \alpha$ and $h_{3}=|y|$. These parameters satisfy the assumptions of Theorem 2 .

Now we are able to compute $B$. Indeed, we have obviously

$$
\begin{aligned}
B & =\max \left\{\frac{q}{\log a_{1}}+\frac{1}{\log |y|}, \frac{q}{k \log \alpha}+\frac{n}{\log |y|}\right\} \\
& =\frac{q}{k \log \alpha}+\frac{n}{\log |y|}<\frac{2 q}{\log \alpha}-\frac{\log \left(3\left|g_{1}\right| / 4\right)}{\log \alpha \log |y|} .
\end{aligned}
$$

Further

$$
Z_{0}=\max \{7+3 \log 3, \log k !\} \leq 14 \log (k !) .
$$

Now we have to distinguish two cases according to the value of $H_{0}$. Assume first that $B \leq(k !)^{14}$. Then $H_{0}=4 m Z_{0}=168 \log (k !)$, which yields

$$
U_{0}<2352(k !)^{7} \log (k !) \log a_{1} \log \alpha \log |y|
$$

hence

$$
\log \left|\Lambda_{1}\right|>-8.64 \cdot 10^{15}(k !)^{7} \log (k !) \log a_{1} \log \alpha \log |y| .
$$

Comparing this inequality with (7), dividing by $(1-\delta) \log |y|$ and using (6) we obtain the upper bound

$$
q<\frac{1}{1-\delta} 8.64 \cdot 10^{15}(k !)^{7} \log (k !) \log a_{1} \log \alpha+\frac{\log \left(1.15 c_{1}\right)}{(1-\delta) \log 2},
$$

which is obviously smaller than $c_{2}$. Hence the assertion is proved in this case. 
Now we turn to the other case $B>(k !)^{14}$, when $H_{0}=\log B$. We have in this case

which yields

$$
U_{0}<(k !)^{7} \log a_{1} \log \alpha \log |y| \log B
$$

$$
\log \left|\Lambda_{1}\right|>-3.68 \cdot 10^{12}(k !)^{7} \log a_{1} \log \alpha \log |y| \log B .
$$

Comparing this inequality with (7), dividing by $(1-\delta) \log \alpha \log |y|$ and using (6) we obtain the inequality

$$
X<\frac{1}{1-\delta} 7.36 \cdot 10^{12}(k !)^{7} \log a_{1} \log X+\frac{2 \log \left(1.15 c_{1}\right)}{(1-\delta) \log \alpha}+\frac{\log \left(25\left|g_{1}\right| / 12\right)}{\log \alpha \log |y|},
$$

where

$$
X=\frac{2 q}{\log \alpha}-\frac{3\left|g_{1}\right| / 4}{\log \alpha \log |y|} .
$$

Application of Lemma 2 yields the upper bound

$$
\begin{aligned}
X< & \frac{4 \log \left(1.15 c_{1}\right)}{(1-\delta) \log \alpha}+\frac{1}{1-\delta} 14.72 \cdot 10^{12}(k !)^{7} \log a_{1} \\
& \left(217 \log (k !) \log \log a_{1}-\log (1-\delta)\right)+\frac{2 \log \left(25\left|g_{1}\right| / 12\right)}{\log \alpha \log |y|},
\end{aligned}
$$

which implies

$$
\begin{aligned}
q< & \frac{2 \log \left(1.15 c_{1}\right)}{1-\delta}+\frac{1}{1-\delta} 7.36 \cdot 10^{12}(k !)^{7} \log a_{1} \log \alpha . \\
& \left(217 \log (k !) \log \log a_{1}-\log (1-\delta)\right)+3 \log \left(5\left|g_{1}\right| / 4\right)
\end{aligned}
$$

The number on the right hand side is again smaller than $c_{2}$, hence the theorem is proved.

To have an impression how large is $c_{2}$ we computed it for the Fibonacci sequence.

Corollary 1. If $F_{n}=y^{q}$ for some $n, y, q \in \mathbb{Z}$ with $|y|, q>1$ then

$$
q<5.1 \cdot 10^{17} \text {. }
$$

Proof. It is well known that

$$
F_{n}=\frac{1}{\sqrt{5}}\left[\left(\frac{1+\sqrt{5}}{2}\right)^{n}-\left(\frac{1-\sqrt{5}}{2}\right)^{n}\right]
$$

Thus the conditions of Theorem 4 are fulfilled and we have to compute $c_{2}$ with the following values of the parameters: $k=2, \alpha=(1+\sqrt{5}) / 2, \delta=-1, c_{1}=1, a_{1}=e^{e}$ and $g_{1}=1 / \sqrt{5}$, which yields the upper bound $5.1 \cdot 10^{17}$ for $q$.

The bound for the exponent of possible perfect powers in the Fibonacci sequence is fairly large. Remark that applying Theorem 2 directly to the linear form (7) coming from the Fibonacci sequence we could be able to improve slightly the bound for $q$, but even this bound would not be smaller then $10^{14}$. The reason is the dependence of Waldschmidt's bound on the parameter $m$.

If, however $g_{1}=1$, which appears for example in the Lucas sequence, we have only two terms in (7) and may use Theorem 3, which yields a much better bound. We do this more generally for the sequences $V_{n}\left(A_{1}, A_{2}\right)$ which is defined by $V_{0}\left(A_{1}, A_{2}\right)=2, V_{1}\left(A_{1}, A_{2}\right)=A_{1}$ and by the recursion $V_{n+2}\left(A_{1}, A_{2}\right)=$ $A_{1} V_{n+1}\left(A_{1}, A_{2}\right)+A_{2} V_{n}\left(A_{1}, A_{2}\right)$ for $n \geq 0$. 
Theorem 5. Let $A_{1}, A_{2}$ be integers such that $A_{1}>0,\left|A_{2}\right|=1$ and $D:=A_{1}^{2}+4 A_{2}>$ 0 . Let further $\alpha=\left(A_{1}+\sqrt{D}\right) / 2$. If the integers $n, y, q$ with $|y|, q>1$ solve the equation

$$
V_{n}\left(A_{1}, A_{2}\right)=y^{q}
$$

then

$$
q<55125 \log \alpha
$$

Moreover, if $\left(A_{1}, A_{2}\right)=(1,-1)$ or $(2,-1)$ then $q<13222$.

Proof. Putting $\beta=\left(A_{1}-\sqrt{D}\right) / 2$, then $V_{n}=\alpha^{n}+\beta^{n}$ holds for all $n$. Assume that the integers $n, y, q$ with $|y|, q>1$ are solutions of (8). As the assertion of the theorem trivially holds for $n \leq 10$, we assume $n>10$ in the sequel and obtain

$$
\left|\frac{y^{q}}{\alpha^{n}}-1\right|=\alpha^{-2 n}<0.01
$$

This implies the inequalities

$$
\begin{aligned}
q \log y-0.01<n \log \alpha & <q \log y+0.01 \\
|\Lambda|:=|q \log y-n \log \alpha| & <1.1 \alpha^{-2 n} .
\end{aligned}
$$

We are ready to apply Theorem 3 to get a lower bound for $|\Lambda|$.

Set $b_{1}=q, b_{2}=n, h_{1}=y$ and

$$
h_{2}=\left\{\begin{array}{rll}
(\log \alpha) / 2 & , \text { if } & A_{1} \geq 3 \\
1 / 2 & \text {, if } & A_{2}=1 \text { and } A_{1}=1,2 .
\end{array}\right.
$$

Then we have in the general case

$$
b=\frac{q}{\log \alpha}+\frac{n}{2 \log y} \leq \frac{3 q}{2 \log \alpha}+0.15
$$

and in the special cases $b<2.04 q+0.015$.

Assuming $q>55000 \log \alpha(q>13000$ in the special cases $)$ we have

$$
\max \{\log b, 10.5,0.5\}=\log b .
$$

In the rest we are dealing with the general case, the proof for the special cases is completely similar. Now Theorem 3 yields

$$
\log |\Lambda| \geq 247.2 \log ^{2}\left(\frac{1.725 q}{\log \alpha}+0.018\right) \log y \log \alpha .
$$

Comparing this with the upper bound we obtain

$$
2 q \log y-0.1<247.2 \log ^{2}\left(\frac{1.725 q}{\log \alpha}+0.018\right) \log y \log \alpha .
$$

Dividing this inequality by $2 \log y \log \alpha / 1.725$ and putting $X:=\frac{1.725 q}{\log \alpha}+0.018$ we have

$$
X<426.5 \log ^{2} X+0.24
$$

which implies $X<95100$ by Lemma 1 and the assertion follows from the definition of $X$.

Even the bound 13222 is too large to try to compute with known techniques all perfect powers in the Lucas sequence. The only recursive sequence for which we know all perfect powers is the Pell sequence $U_{n}(2,1)$. Pethö [P5] and independently Cohn $[\mathrm{Co}]$ proved that the equation

$$
P_{n}=y^{q}
$$


has solutions only for $q=2$ and these are $(n, y)=(0,0),(1,1)$ and $(7,13)$. Pethö ruled out the odd exponents by elementary means. For $q=2$ and $n$ odd Wolfskill [Wo] proved that $n<469$, and below this bound is very easy to find all squares. The statement for even index squares follows from divisibility properties of the Pell numbers.

2.2. Perfect powers in high order recurrences. If $G_{n}$ is a LRS satisfying the conditions of Theorem 4 then there are no non-trivial (from 0 and 1 different) $q$-th power in $G_{n}$ provided $q$ is large enough. You have seen, that this bound is usually large. But this is not so bad, because the constants in the linear form bounds are continuously improved and I expect in the future for a wide class of LRS at least such a good bound as for the Lucas sequence.

Four years ago I continued with the following sentences: Annoying is that we have generaly no information about small powers. The tribonacci sequence defined in the Problem of [P6] satisfies the condition of Theorem 4, but we do not know how many squares, third powers, etc. appear in it. We are not able to prove even that their number is finite.

Similarly, we know nothing about the squares or higher powers in the sequence $\left(2^{n}-1\right)\left(3^{n}-1\right)$. This seems hard for squares and a bit easier for higher powers.

The situation changed considerably in the recent years. L. Szalay [Sz] established all squares in the sequence $\left(2^{n}-1\right)\left(3^{n}-1\right)$. Hajdu and Szalay [HSz] did the same for the sequences $\left(2^{n}-1\right)\left(6^{n}-1\right)$ and $\left(a^{n}-1\right)\left(a^{k n}-1\right)$. In both papers there are used only elementary methods.

Much more interesting are the results of Corvaja and Zannier [CZ1] and [CZ2]. They considered in [CZ1] LRS with integer characteristic roots and proved: Let

$$
G_{n}=g_{1} a_{1}^{n}+\cdots+g_{k} a_{k}^{n},
$$

where $k \geq 2, g_{1}, \ldots, g_{k}$ are non-zero algebraic numbers and $a_{1}>a_{2}>\cdots>a_{k}>0$ are integers with $a_{1}, a_{2}$ coprime. Then for every integer $d \geq 2$ equation (3) has only finitely many solutions $n, y \in \mathbb{N}$. Remark that they used in the proof W.M. Schmidt's [Schm] celebrated subspace theorem, hence the result is not effective.

Combining this result with Theorem 4 one obtains under the same assumptions as above that there are only finitely many perfect powers in the sequence $G_{n}$.

Moreover combining Theorem 4 with Theorem 2 of [CZ2] we are able to prove the following theorem.

Theorem 6. Let $G_{n}$ be a third order LRS. Assume that the characteristic polynomial of $G_{n}$ is irreducible and has a dominating root. Then there are only finitely many perfect powers in $G_{n}$.

Remark 1. The tribonacci numbers are defined by the initial terms $T_{0}=T_{1}=$ $0, T_{2}=1$ and by the recursion $T_{n+3}=T_{n+2}+T_{n+1}+T_{n}$ for $n \geq 0$. In Part I [P6] I asked whether $T_{0}=T_{1}=0, T_{2}=T_{3}=1, T_{5}=4, T_{10}=81, T_{16}=3136=56^{2}$ and $T_{18}=10609=103^{2}$ are the only perfect squares among the tribonacci numbers. It follows from Theorem 6 that there are only finitely many perfect powers in $T_{n}$. This is a partial answer to my question. Remark that Theorem 6 is not effective, hence we have presently no algorithm for computing all powers in the sequence $T_{n}$.

Proof of Theorem 6. The assumptions of Theorem 4 fulfill, hence if $G_{n}=y^{q}$ and $|y|>1$ then $q<c_{2}$ with an effectively computable constant $c_{2}$.

In the rest of the proof let $2 \leq q<c_{2}$ be fixed. Assume that (3) has for this $q$ infinitely many solutions in $n, y \in \mathbb{N}, y>1$. Then there exist by [CZ2] (Theorem 2) non-zero algebraic numbers $d_{1}, \ldots, d_{r}, \delta_{1}, \ldots, \delta_{r}$ such that

$$
G_{n}=g_{1} \alpha_{1}^{n}+g_{2} \alpha_{2}^{n}+g_{3} \alpha_{3}^{n}=\left(d_{1} \delta_{1}^{n}+\cdots+d_{r} \delta_{r}^{n}\right)^{q}
$$


holds for all elements $n$ of an arithmetical progression $P$. We may assume without loss of generality that $\left|\delta_{1}\right| \geq \cdots \geq\left|\delta_{r}\right|$.

There exist, by a theorem of van der Poorten and Schlickewei [PSch], for any $\varepsilon>0$ constants $c_{4}$ and $c_{5}(\varepsilon)$ such that

$$
c_{5}(\varepsilon)\left|\delta_{1}\right|^{n(1-\varepsilon)} \leq\left|\sum_{i=1}^{r} d_{i} \delta_{i}^{n}\right| \leq c_{4}\left|\delta_{1}\right|^{n}
$$

holds for all $n>n(\varepsilon)$. On the other hand

$$
\left|g_{1}\right|\left|\alpha_{1}\right|^{n} / 2 \leq\left|G_{n}\right| \leq 2\left|g_{1}\right|\left|\alpha_{1}\right|^{n}
$$

is true, provided $n$ is large enough.

Hence on one hand

$$
\left|\frac{\delta_{1}^{q}}{\alpha_{1}}\right|^{n} \geq \frac{\left|g_{1}\right|}{2 c_{4}^{q}}
$$

for all sufficiently large $n$, which implies

$$
\left|\alpha_{1}\right| \leq\left|\delta_{1}\right|^{q} .
$$

On the other hand we have

$$
\left(\frac{\left|\delta_{1}^{q}\right|^{1-\varepsilon}}{\left|\alpha_{1}\right|}\right)^{n} \geq \frac{2\left|g_{1}\right|}{\left(c_{5}(\varepsilon)\right)^{q}}
$$

for any fixed $\varepsilon>0$ and all $n \geq n(\varepsilon)$. This implies

$$
\left|\alpha_{1}\right| \geq\left|\delta_{1}\right|^{q(1-\varepsilon)}
$$

for all $\varepsilon>0$. The lower and upper bounds for $\left|\alpha_{1}\right|$ imply $\left|\delta_{1}\right|=\left|\alpha_{1}\right|^{1 / q}$.

Assume that $\left|\delta_{1}\right|=\cdots=\left|\delta_{s}\right|=\left|\alpha_{1}\right|^{1 / q}>\left|\delta_{s+1}\right| \geq\left|\delta_{r}\right|$. Define $D_{1}(n)=$ $\sum_{i=1}^{s} d_{i} \delta_{i}^{n}$ and $D_{2}(n)=\sum_{i=s+1}^{r} d_{i} \delta_{i}^{n}$ and rewrite (9) as

$$
g_{1} \alpha_{1}^{n}+g_{2} \alpha_{2}^{n}+g_{3} \alpha_{3}^{n}=\left(D_{1}(n)+D_{2}(n)\right)^{q}=D_{1}(n)^{q}+\sum_{j=1}^{q}\left(\begin{array}{l}
q \\
j
\end{array}\right) D_{1}(n)^{q-j} D_{2}(n)^{j} .
$$

This implies

$$
g_{1} \alpha_{1}^{n}-D_{1}(n)^{q}=\sum_{j=1}^{q}\left(\begin{array}{l}
q \\
j
\end{array}\right) D_{1}(n)^{q-j} D_{2}(n)^{j}-g_{2} \alpha_{2}^{n}-g_{3} \alpha_{3}^{n} .
$$

There is staying on the left hand side a finite power sum. If it is non-zero then for any $\varepsilon>0$ its absolute value is bounded below by $c_{6}(\varepsilon)\left|\alpha_{1}\right|^{n(1-\varepsilon)}$. In contrast the right hand side is obviously bounded above by $c_{7}\left|\alpha_{1}\right|^{n(q-1) / q}<c_{7}\left|\alpha_{1}\right|^{n(1-\eta)}$ with a fixed $\eta>0$. This is a contradiction, hence

$$
g_{1} \alpha_{1}^{n}=D_{1}(n)^{q}
$$

which can be written as

$$
g_{1}^{1 / q}\left(\alpha_{1}^{1 / q}\right)^{n}-\sum_{i=1}^{s} d_{i} \delta_{i}^{n}=0 .
$$

This is an $S$-unit equation in the field $\mathbb{Q}\left(g_{1}^{1 / q}, \alpha_{1}^{1 / q}, d_{1}, \ldots, d_{s}, \delta_{1}, \ldots, \delta_{s}\right)$, which has infinitely many solutions in $n \in \mathbb{N}$. By the theorem of Evertse [Ev1] there exists a $1 \leq i \leq s$ such that $g_{1}^{1 / q}\left(\alpha_{1}^{1 / q}\right)^{n}=d_{i} \delta_{i}^{n}$ holds infinitely often. We may assume $i=1$ without loss of generality, i.e. we have

$$
g_{1}^{1 / q}\left(\alpha_{1}^{1 / q}\right)^{n}=d_{1} \delta_{1}^{n} \quad \text { and } \quad \sum_{i=2}^{s} d_{i} \delta_{i}^{n}=0
$$

for infinitely many $n \in \mathbb{N}$. 
For such integers $n$ we obtain

$$
\sum_{j=1}^{q}\left(\begin{array}{l}
q \\
j
\end{array}\right) d_{1}^{q-j} \delta_{1}^{(q-j) n} D_{2}(n)^{j}-g_{2} \alpha_{2}^{n}-g_{3} \alpha_{3}^{n}=0
$$

from equation (10). As $\alpha_{2} / \alpha_{3}$ is not a root of unity the sum $g_{2} \alpha_{2}^{n}+g_{3} \alpha_{3}^{n}$ never vanish if $n$ is large enough, hence $D_{2}(n) \neq 0$ for such $n$ 's. The function

$$
\left(\begin{array}{l}
q \\
j
\end{array}\right) d_{1}^{q-j} \delta_{1}^{(q-j) n} D_{2}(n)^{j}
$$

is for fixed $n$ strictly decreasing in $j$, hence writing it as a sum of exponential functions it has at least $q$ summands such that no proper subsums of their sum vanishes for infinitely many $n$. From these one can be equal to $g_{2} \alpha_{2}^{n}$ and an other equal to $g_{3} \alpha_{3}^{n}$, hence if $q>2$ then (11) cannot hold for infinitely many $n$.

Finally let $q=2$. Then (11) simplifies to

$$
2 d_{1} \delta_{1}^{n}\left(\sum_{i=2}^{r} d_{i} \delta_{i}^{n}\right)+\left(\sum_{i=2}^{r} d_{i} \delta_{i}^{n}\right)^{2}-g_{2} \alpha_{2}^{n}-g_{3} \alpha_{3}^{n}=0 .
$$

This equation has infinitely many solutions in $n$. As $\sum_{i=2}^{r} d_{i} \delta_{i}^{n} \neq 0$ for all large enough $n$, there exists a minimal index $2 \leq i_{0} \leq r$ and $J \subseteq R=\{2, \ldots, r\}$ such that $i_{0} \in J$,

$$
\sum_{j \in R \backslash J} d_{j} \delta_{j}^{n}=0 \quad \text { and } \sum_{j \in J_{1} \subseteq J} d_{j} \delta_{j}^{n} \neq 0
$$

for infinitely many solutions of (12) and for all $\emptyset \neq J_{1} \subseteq J$. Therefore $|J|=1$ and

$$
2 d_{1} \delta_{1}^{n} d_{i_{0}} \delta_{i_{0}}^{n}+d_{i_{0}}^{2} \delta_{i_{0}}^{2} n-g_{2} \alpha_{2}^{n}-g_{3} \alpha_{3}^{n}=0 .
$$

Hence the system of equations

$$
\begin{aligned}
2 d_{1} \delta_{1}^{n} d_{i_{0}} \delta_{i_{0}}^{n} & =g_{2} \alpha_{2}^{n} \\
d_{i_{0}}^{2} \delta_{i_{0}}^{2} n & =g_{3} \alpha_{3}^{n}
\end{aligned}
$$

or the analogous system of equations, where the right hand sizes are interchanged, has infinitely many solutions in $n$.

These implies $\delta_{1} \delta_{i_{0}}=\alpha_{2} \zeta_{2}$ and $\delta_{i_{0}}^{2}=\alpha_{3} \zeta_{3}$, with some roots of unity $\zeta_{2}, \zeta_{3}$. We also have $\delta_{1}^{2}=\alpha_{1} \zeta_{1}$ with a root of unity $\zeta_{1}$. These three relations imply

$$
\frac{\alpha_{2}^{2}}{\alpha_{1} \alpha_{3}}=\frac{\zeta_{1} \zeta_{3}}{\zeta_{2}^{2}} .
$$

Using finally that $\alpha_{1} \alpha_{2} \alpha_{3}$ is an integer $A$, we conclude that $\alpha_{2}^{3}=A \zeta$, where $\zeta$ is a root of unity. Taking conjugates we conclude that $\left|\alpha_{1}\right|=\left|\alpha_{2}\right|=\left|\alpha_{3}\right|$, which contradicts the assumption that one of the roots is dominating. The Theorem is proved.

2.3. Perfect powers in second order recurrences. For second order recurrences we can bound effectively not only the exponents of the perfect powers appearing in the sequence, but also the largest index for which a term of the sequence can be a perfect power. This was proved by Shorey and Stewart [ShSt1] and independently by me $[\mathrm{P} 1]$.

Theorem 7. Let $G_{n}$ be a non-degenerated second order $L R S$ and $d \in \mathbb{Z}$. Then there exist effectively computable positive constants $c_{1}, c_{2}$ depending only on $G_{0}, G_{1}, A_{1}$, $A_{2}$ and on $d$ such that if for the integers $n, y, q$ such that $q>1$ the equation

$$
G_{n}=y^{q}
$$

holds, then:

(a) If $|y|>1$, then $\max \{|y|, n, q\}<c_{1}$, 
(b) if $|y| \leq 1$, then $n<c_{2}$.

Proof. We may assume by Theorem 4, that if $|y|>1$, then $q$ is bounded by an effectively computable positive constants $c_{3}$ depending only on $G_{0}, G_{1}, A_{1}, A_{2}$ and on $d$. Let fix $q$ such that $1<q<c_{3}$.

Write

and let

$$
G_{n}=g_{1} \alpha_{1}^{n}+g_{2} \alpha_{2}^{n}
$$

$$
H_{n}=\frac{g_{1} \alpha_{1}^{n}-g_{2} \alpha_{2}^{n}}{\alpha_{1}-\alpha_{2}} .
$$

It is easy to check that $H_{n}$ is also an LRS of integers, $G_{n}$ and $H_{n}$ have the same characteristic polynomial and

$$
G_{n}^{2}-\left(\alpha_{1}-\alpha_{2}\right)^{2} H_{n}^{2}=4\left(\alpha_{1} \alpha_{2}\right)^{n} .
$$

Remark that $\left(\alpha_{1}-\alpha_{2}\right)^{2}=A_{1}^{2}+4 A_{2}$ and $\alpha_{1} \alpha_{2}=-A_{2}$ are integers. Let $S$ be the set of all prime divisors of $A_{2}$ and $n, y$ be a solution of (13) for our fixed $q$ with $|y|>1$. There exist integers $r, s$ such that $n=s(2 q)+r$ and $0 \leq r<2 q$. The rational numbers $\frac{G_{n}}{\left(A_{2}\right)^{q s}}$ and $\frac{y}{s}$ are $S$-integral solutions of the hyperelliptic equation

$$
z^{2}=\left(A_{1}^{2}+4 A_{2}\right) u^{2 q}+4\left(-A_{2}\right)^{r}
$$

The polynomial staying on the right hand side of this equation has for all $0 \leq r<2 q$ at least three simple zeros, hence it has only finitely many effectively computable S-integer solutions.

As there exists only finitely many possibilities for $q$ and for $r$ the number of the hyperelliptic equations to be considered is finite, thus the number of solutions is finite and effectively computable.

The rest of the proof is easy, you find details in [P1, ShSt1] or in [ShT].

2.4. A method for determining squares in second order LRS. The above proof is simple and is very well for theoretical purposes. Its drawback is that it is hard to find power values in given LRS following its line, because we transform the problem to finitely many hyperelliptic equations and their solution is not at all easy.

Mignotte and Pethő [MP2] proposed a more direct way to solve (13) in case $q=2$ and $\left|A_{2}\right|=1$. We present now their method in this special form. They remarked that the method can be generalized to arbitrary $A_{2}$ and for arbitrary $q$.

Let $G_{n}$ be a second order recursive sequence with $\left|A_{2}\right|=1, d \in \mathbb{Z}$ and consider the equation

$$
G_{n}=g_{1} \alpha_{1}^{n}+g_{2} \alpha_{2}^{n}=d y^{2} .
$$

As $\left|A_{2}\right|=1$ the numbers $\alpha_{1}, \alpha_{2}$ are real quadratic units. Put $\mathbb{K}=\mathbb{Q}\left(\alpha_{1}\right)$. Let $\gamma^{\prime}$ denotes the conjugate of $\gamma \in \mathbb{K}$. Then $\alpha_{2}=\alpha_{1}{ }^{\prime}$. We may assume without loss of generality that $\alpha_{1}>\left|\alpha_{2}\right|$. Adjusting our equation appropriately (changing $g_{1}$ and $g_{2}$ with $\alpha_{1} g_{1}$ and $\alpha_{2} g_{2}$ respectively if $\mathrm{n}$ is odd; multiplying the equation with the square free part of $g_{2}$ and with -1 if necessary) we see that it is enough to deal with the equation

$$
a \alpha_{1}^{2 m}-b^{2} \alpha_{2}^{2 m}=c y^{2},
$$

where $a, b$ and $c \in \mathbb{Z}_{\mathbb{K}}$ and $m, y \geq 0$ are integers. Our aim is to prove an upper bound for $m$.

Let $\mathbb{L}=\mathbb{K}(\sqrt{-c})$ and assume that $\mathbb{L}$ is a quadratic extension of $\mathbb{K}$, i.e. $[\mathbb{L}: \mathbb{Q}]=$ 4. Then our equation implies

$$
N_{\mathbb{L} / \mathbb{Q}}\left(b \alpha_{2}^{m}+\sqrt{-c} y\right)=N_{\mathbb{L} / \mathbb{Q}}(a)=A,
$$


with an integer $A$.

Choose in $\mathbb{Z}_{\mathbb{L}}$, in the ring of integers of $\mathbb{L}$, units $\eta_{2}, \ldots, \eta_{r} ; r=1,2$ or 3 such that the group $\mathbf{U}$ generated by $\eta_{1}=\alpha, \eta_{2}, \ldots, \eta_{r}$ has finite index in the group of units of $\mathbb{Z}_{\mathbb{L}}$. There exists in $\mathbb{Z}_{\mathbb{L}}$ a maximal finite set of, with respect to $\mathbf{U}$, non-associated elements of norm $A$. This set will be denoted by $\mathbf{A}$. Then there exist for all $m, x \in \mathbb{Z}$ with (14) a $\gamma \in \mathbf{A}$ and $\varepsilon \in \mathbf{U}$ such that

$$
b \alpha_{2}^{m}+\sqrt{-c} y=\gamma \varepsilon .
$$

Let order the conjugates $\mathbb{L}^{(i)}, i=1,2,3,4$ of $\mathbb{L}$ according the following ordering of the conjugates of $\sqrt{-c}: \sqrt{-c},-\sqrt{-c}, \sqrt{-c^{\prime}},-\sqrt{-c^{\prime}}$. Let $R$ denote the regulator of $\mathbf{U}$, i.e. the absolute value of the determinant of the matrix $\left(\log \left|\eta_{i}^{(j)}\right|\right)_{1 \leq i, j \leq r}$ and finally, for $\delta \in \mathbb{Z}_{\mathbb{K}}$ let

$$
\overline{|\delta|}:=\max \left\{\mid \delta^{(i)}, i=1, \ldots, 4\right\} .
$$

It is easy to see that if $m>m_{0}$ then

$$
\frac{1}{2} \frac{\sqrt{|a|}}{\left|\gamma^{(i)}\right|} \alpha_{1}^{m}<\left|\varepsilon^{(i)}\right|<\frac{2 \sqrt{|a|}}{\left|\gamma^{(i)}\right|} \alpha_{1}^{m}
$$

for $i=1,2$; and if $b^{\prime}>0$, which we may assume without loss of generality, then

$$
\frac{b^{\prime}}{2\left|\gamma^{(3)}\right|} \alpha_{1}^{m}<\left|\varepsilon^{(3)}\right|<2 \frac{b^{\prime}}{\left|\gamma^{(3)}\right|} \alpha_{1}^{m}
$$

and

$$
\frac{\left|a^{\prime}\right|}{2 b^{\prime}\left|\gamma^{(4)}\right|} \alpha_{1}^{-3 m}<\left|\varepsilon^{(4)}\right|<\frac{2\left|a^{\prime}\right|}{b^{\prime}\left|\gamma^{(4)}\right|} \alpha_{1}^{-3 m}
$$

hold. We remark that if $b^{\prime}<0$ then only the role of $\varepsilon^{(3)}$ and $\varepsilon^{(4)}$ changes.

The last inequalities imply that if $c^{\prime}>0$ then (14) has only finitely many solutions and they are very easy to compute. In fact $\varepsilon^{(3)}$ and $\varepsilon^{(4)}$ are in this case conjugate complex numbers, hence

$$
\frac{b^{\prime}}{2\left|\gamma^{(3)}\right|} \alpha_{1}^{m}<\left|\varepsilon^{(3)}\right|=\left|\varepsilon^{(4)}\right|<\frac{2\left|a^{\prime}\right|}{b^{\prime}\left|\gamma^{(4)}\right|} \alpha_{1}^{-3 m},
$$

i.e $m<\frac{1}{4} \log \left|\frac{4 a^{\prime} \gamma^{(3)}}{b^{\prime 2} \gamma^{(4)}}\right|$.

The situation is more interesting when $c^{\prime}<0$. Then $\varepsilon^{(3)}$ and $\varepsilon^{(4)}$ are real numbers and we will use estimations on linear forms in logarithms of algebraic numbers to establish an upper bound for $m$.

Let first $c>0$ (and $\left.c^{\prime}<0\right)$. Then $\mathbb{L}$ has two nonreal and two real conjugates, and there exist $u_{1}, u_{2} \in \mathbb{Z}$ with $\varepsilon=\eta_{1}^{u_{1}} \eta_{2}^{u_{2}}$. The estimations (16) with $i=2$ and (17) yield

$$
\left|u_{1}\right|<\frac{2 m \log \alpha_{1}|\log | \eta_{2}^{(3)}|-\log | \eta_{2}^{(2)}||}{R}+c_{1}
$$

and

where

$$
\left|u_{2}\right|<\frac{4 m \log ^{2} \alpha_{1}}{R}+c_{1}
$$

$$
c_{1}=2 \log \left(3 \overline{|a|\left|b^{2}\right|} \overline{\left|\frac{1}{\gamma}\right|}\right) \max \left\{\log \alpha_{1}, \log \overline{\left|\eta_{2}\right|}\right\} / R .
$$

We have

$$
\gamma^{(1)} \varepsilon^{(1)}+\gamma^{(2)} \varepsilon^{(2)}=2 b \alpha_{2}^{m},
$$


hence

$$
\left|1+\frac{\gamma^{(2)}}{\gamma^{(1)}}\left(\frac{\eta_{2}^{(2)}}{\eta_{2}^{(1)}}\right)^{u_{2}}\right|<\frac{4 b}{\sqrt{|a|}} \alpha_{1}^{-2 m}
$$

If $m>m_{0}$, then $\frac{4 b}{\sqrt{|a|}} \alpha_{1}^{-2 m}<\frac{1}{2}$ and so

$$
\left|\Lambda_{1}\right|=\left|\log \left(-\frac{\gamma^{(2)}}{\gamma^{(1)}}\right)+u_{2} \log \left(\frac{\eta_{2}^{(2)}}{\eta_{2}^{(1)}}\right)+u_{0} \pi\right|<\frac{4.1|b|}{\sqrt{|a|}} \alpha_{1}^{-2 m},
$$

where $u_{0} \in \mathbb{Z}$ and we take the principal value of the complex logarithm function, i.e. $-\pi \leq \log (z) \leq \pi$ for every $z \in \mathbb{C}$. The last inequality yields $\left|u_{0}\right|<\left|u_{2}\right|+2$.

As the field $\mathbb{Q}\left(\gamma^{(2)} / \gamma^{(1)}, \eta_{2}^{(2)} / \eta_{2}^{(1)}, \sqrt{-1}\right)$ is obviously a subfield of

$$
\mathbb{Q}\left(\sqrt{-c}, \sqrt{-c^{\prime}} \sqrt{-1}\right)
$$

which is of degree at most 16 over $\mathbb{Q}$ we can set $D=16$ and $B=\left|u_{2}\right|+2$ in Theorem 1 which yields the lower bound

$$
\left|\Lambda_{1}\right|>\exp \left\{-1.25 \cdot 10^{18} h\left(\frac{\gamma^{(2)}}{\gamma^{(1)}}\right) h\left(\frac{\eta_{2}^{(2)}}{\eta_{2}^{(1)}}\right) \log \left(\left|u_{2}\right|+2\right)\right\} .
$$

Comparing the lower and upper bounds for $\left|\Lambda_{1}\right|$ we conclude

$$
\begin{aligned}
& 2 m \log \alpha_{1}-\log \frac{4.1|b|}{\sqrt{|a|}}< \\
& 1.25 \cdot 10^{18} h\left(\frac{\gamma^{(2)}}{\gamma^{(1)}}\right) h\left(\frac{\eta_{2}^{(2)}}{\eta_{2}^{(1)}}\right) \log \left(\frac{4 m \log ^{2} \alpha}{R}+c_{1}+8\right) .
\end{aligned}
$$

This inequality yields an upper bound for $m$, which we shall only compute knowing the actual values of the occuring parameters.

Let now $c<0$ (and $c^{\prime}<0$ ). Then all conjugates of $\mathbb{L}$ are real and there exist $u_{1}, u_{2}, u_{3} \in \mathbb{Z}$ with $\varepsilon=\eta_{1}^{u_{1}} \eta_{2}^{u_{2}} \eta_{3}^{u_{3}}$. We recall $\eta_{1}=\alpha_{1}$. The estimations (16) with $i=1,2$ and (17) yield

$$
\left|u_{i}\right|<\frac{4 m \log ^{2} \alpha_{1} \log h}{R}+c_{2}, i=2,3
$$

where

$$
c_{2}=3 \sqrt{3} \log \left(3 \overline{|a|\left|b^{2}\right| \mid 1 / \gamma}\right) \log \alpha_{1} \log \overline{\left|\eta_{2}\right|} \log \overline{\left|\eta_{3}\right|} / R
$$

and $h=\max \left\{\overline{\left|\eta_{2}\right|}, \overline{\left|\eta_{3}\right|}\right\}$.

Similarly to the above case, but working with real instead of complex logarithms we get

$$
\left|\Lambda_{2}\right|=|\log | \frac{\gamma^{(2)}}{\gamma^{(1)}}\left|+u_{2} \log \right| \frac{\eta_{2}^{(2)}}{\eta_{2}^{(1)}}\left|+u_{3} \log \right| \frac{\eta_{3}^{(2)}}{\eta_{3}^{(1)}}||<\frac{5.6|b|}{\sqrt{|a|}} \alpha_{1}^{-2 m} .
$$

The parameters in the application of Theorem 1 are the same as earlier except that $D=8$ and $B=\max \left\{\left|u_{2}\right|,\left|u_{3}\right|\right\}$. Hence Theorem 1 implies

$$
\begin{aligned}
& 2 m \log \alpha_{1}-\log \frac{5.6|b|}{\sqrt{|a|}}< \\
& \quad 3.87 \cdot 10^{16} h\left(\frac{\gamma^{(2)}}{\gamma^{(1)}}\right) h\left(\frac{\eta_{2}^{(2)}}{\eta_{2}^{(1)}}\right) h\left(\frac{\eta_{3}^{(2)}}{\eta_{3}^{(1)}}\right) \log \left(\frac{4 m \log ^{2} \alpha_{1} \log h}{R}+c_{2}\right) .
\end{aligned}
$$

We again do not express an upper bound for $m$ explicitly, but rather show through an example how to use inequalities (19) and (20). 
Example Let $G_{0}=0, G_{1}=10$ and $G_{n+2}=10 G_{n+1}-G_{n}$ and consider the equation $G_{2 m+1}=\square$. It is easy to see, that if we are able to solve this equation then we can solve $G_{n}=\square$ too. (See [MP2].)

To solve the equation $G_{2 m+1}=\square$ we intend to apply the method described above. First we compute the necessary initial data. We have $\alpha_{1}=5+2 \sqrt{6}$, $\alpha_{2}=5-2 \sqrt{6}$ and $c=-12+5 \sqrt{6}$, thus our equation has the form

$$
\alpha_{1}^{2 m}-\alpha_{2}^{2} \alpha_{2}^{2 m}=4 \alpha_{2} \sqrt{6} w^{2}=4(5 \sqrt{6}-12) w^{2} .
$$

It is easy to see that it has only one solution $(m, w)=(0,1)$ in the range $0 \leq m \leq 10$. If $m>10$ then (16) is obviously true. Thus we may assume in the sequel $m>10$.

The algebraic number field $\mathbb{L}=\mathbb{Q}(\sqrt{6}, \sqrt{12-5 \sqrt{6}})$, has two real and two nonreal conjugates. The units $\alpha_{1}$ and $\eta_{2}=5-2 \sqrt{12-5 \sqrt{6}}-2 \sqrt{6}$ are fundamental units in $\mathbb{Z}_{\mathbb{L}}$, hence its regulator is $R=6.83836$ and we get

$$
\left|u_{2}\right|<3.07398 m+8.95847 \text {. }
$$

As $\gamma=1$ there are only two summands in $\Lambda_{1}$, actually it has the form

$$
\Lambda_{1}=\left|u_{2} \log \left(\frac{5-2 \sqrt{6}+2 \sqrt{12-5 \sqrt{6}}}{5-2 \sqrt{6}-2 \sqrt{12-5 \sqrt{6}}}\right)+u_{0} \pi\right|<0.042 \alpha_{1}^{-2 m} .
$$

As we proved, there are generally three logarithms in $\Lambda_{1}$, but in the actual example we have only two, therefore in the, to (14) analogous inequality we get a much better constant. More precisely we have

$$
4.58486 m+3.17387<6.81595 \cdot 10^{11} \log (12.4 m+40),
$$

which implies $m<5 \cdot 10^{12}$ and $\left|u_{2}\right|<1.55 \cdot 10^{13}$. Dividing the inequality for $\Lambda_{1}$ by $u_{2} \pi$ we see that, as $m>10, u_{0} / u_{2}$ is a convergent of

$\log \left(\frac{\eta_{2}^{(2)}}{\eta_{2}^{(1)}}\right) / \pi=\delta=.93557845273700309088141600367180617252445255312155 \ldots$

The denominator of the 26-th convergent of $\delta, \frac{51706546491839}{55266927472061}$, is larger than $10^{14}$, hence by the well known extremality property of the convergents of real numbers we obtain

$$
\left|u_{0}-u_{2} \delta\right| \geq|51706546491839-55266927472061 \cdot \delta|>0.16132 \cdot 10^{-13} .
$$

Comparing this inequality with (21) we obtain

$$
0.16132 \cdot 10^{-13}<0.0134 \alpha_{1}^{-2 m},
$$

which implies $m \leq 5$. Thus our equation has only the trivial solution $(m, w)=(0,1)$.

Acknowledgement. The author thanks very much for the careful work of the referee.

\section{REFERENCES}

[Ba1] A. BAKER, Linear forms in the logarithms of algebraic numbers, Mathematika 13 (1966), $204-216$.

[Ba2] A. BAKeR, Transcendental Number Theory, Cambridge Univ. Press, 1975.

[BW] A. BAKER and G. Wüstholz, Linear forms and group varieties, J. reine und angew. Math. 442 (1993), 19-62.

[Co] J.H.E. Cohn, Perfect Pell powers, Glasgow Math. J. 38 (1996), 19-20.

[CZ1] P. CoRvaJA and U. ZANNIER, Diophantine equations with power sums and universal Hilbert sets, Indag Math., N.S. 9 (1998), 317-332.

[CZ2] P. Corvaja and U. ZANnier, Some new applications of the subspace theorem, manuscript.

[Ev1] J.H. EverTse, On sums of S-units and linear recurrences, Comp. Math. 53 (1984), 225-244. 
[HSz] L. HAJDU and L. SzAlay, On the diophantine equations $\left(2^{n}-1\right)\left(6^{n}-1\right)=x^{2}$ and $\left(a^{n}-\right.$ 1) $\left(a^{k n}-1\right)=x^{2}$, Periodica Math. Hungar., 40 (2000), 141-145.

[K1] P. Kiss, On common terms of linear recurrences, Acta Math. Acad. Sci. Hungar. 40 (1982) 119-123.

[K2] P. KIss, Pure powers and power classes in recurrence sequences, Math. Slovaca, 44 (1994) 525-529.

[K3] P. KIss, Note on a result of I. Nemes and A. Pethö concerning polynomial values in linear recurrences, Publ. Math. Debrecen 56 (2000), 451-455.

[LMN] M. Laurent, M. Mignotte et Y. Nesterenko, Formes linéaires en deux logarithmes et déterminants d'interpolation, J. Number Theory, 55 (1995), 285-321.

[MP1] M. Mignotte and A. Ретнő, Sur les carrés dans certaines suites de Lucas, Journal de Théorie Nombres de Bordeaux, 5 (1993), 333-341.

[MP2] M. Mignotte and A. PEтHö, On the system of diophantine equations $x^{2}-6 y^{2}=-5$ and $x=2 z^{2}-1$, Math. Scandinavica, 76 (1995), 50-60.

[MT] M. Mignotte and N. Tzanakis, Arithmetical study of recurrence sequences, Acta Arith., 57 (1991). 357-364.

[Mo] L.J. Mordell, Diophantine Equations, Academic Press, 1969.

[N] I. Nemes, On the solution of the diophantine equation $G_{n}=P(x)$ with sieve method, in Computational Number Theory, Walter de Gruyter, Berlin-New York, 1991, pp. 303-311.

[NP1] I. Nemes and A. Peтнő, Polynomial values in linear recurrences I., Publ. Math. Debrecen, 31 (1984), 229-233.

[NP2] I. Nemes and A. РетнŐ, Polynomial values in linear recurrences II., J. Number Theory, 24 (1986), 47-53.

[P1] A. Ретно̋, Perfect powers in second order linear recurrences, J. Number Theory, 15 (1982), $5-13$.

[P2] A. Pетнö, Full cubes in the Fibonacci sequence, Publ. Math. Debrecen, 30 (1983), 117-127.

[P3] A. РетнÖ, Perfect powers in second order recurrences, in: Topics in Classical Number Theory, Budapest, 1981, 1217-1227.

[P4] A. PeтHö, On the solution of the equation $G_{n}=P(x)$, in Fibonacci Numbers and Their Applications, D. Reidel Publ. Comp. 1986, 193-201.

[P5] A. Ретнӧ, The Pell sequence contains only trivial perfect powers, in: Proc. Sets Graphs and Numbers. Coll. Math. Soc. János Bolyai Vol. 60. pp 561-568.

[P6] A. Ретно̋, Diophantine properties of linear recursive sequences. I. Bergum, G. E. (ed.) et al., Applications of Fibonacci numbers. Volume 7: Proceedings of the 7th international research conference on Fibonacci numbers and their applications, Graz, Austria, July 15-19, 1996. Dordrecht: Kluwer Academic Publishers. 295-309 (1998).

[PW] A. Pethö and B.M.M. DE Weger, Product of prime powers in binary recurrence sequences I., Math. Comp. 47 (1986), $713-727$.

[PSch] A.J. VAN DER PoORTEN and H.P. SChlickewei, The growth conditions for recurrence sequences, Macquarie Univ. Math. Rep. 82-0041. North Ryde, Australia.

[Schm] W.M. Sснмidt, Linearformen mit algebraischen Koeffizienten II., Math. Annalen, 191 (1972), 525-551.

[ShT] T.N. Shorey and R. Tijdeman, Exponential Diophantine Equations, Cambridge Univ. Press 1986.

[ShSt1] T.N. Shorey and C.L. Stewart, On the Diophantine equation $a x^{2 t}+b x^{t} y+c y^{2}=d$ and pure powers in recurrences, Math. Scand., 52 (1983), 24-36.

[ShSt2] T.N. Shorey and C.L. SteWART, Pure powers in recurrence sequences and some related diophantine equations, J. Number Theory, 27 (1987) 324-352.

[Sm] N.P. Smart, The Algorithmic Resolution of Diophantine Equations, London Math. Soc. Student Text, 41, Cambridge Univ. Press, 1998.

[St] C.L. Stewart, On some diophantine equations and related linear recurrence sequences, Seminaire Delange-Pisot-Poitou 1980-81.

[Sz] L. Szalay, On the diophantine equations $\left(2^{n}-1\right)\left(3^{n}-1\right)=x^{2}$, Publ. Math. Debrecen 57 (2000), 1-9.

[W] M. Waldschmidt, Minorations de combinaisons linéares de logarithmes de nombres algébriques, J. Canad. Math. 45 (1993), 176-224.

[Wo] J. Wolfskill, Bounding squares in second order recurrence sequences, Acta Arith. 54 (1989) 127-145.

Received September 28, 2000; December 4, 2000 in revised form. 
InStitute FOR MATHEMATICS AND INFORMATICS,

University of Debrecen,

H-4010 Debrecen, PO Box 12, Hungary 